\title{
A Study on the Indoor Positioning Method of a Motorcar Detection System Based on CSS (Chirp Spread Spectrum)
}

\author{
Ho-Hung Jung ${ }^{1, ~ *, ~ C h a n g l o n g ~} \mathrm{Li}^{2}$, Key-Seo Lee ${ }^{2}$ \\ ${ }^{1}$ Seoul Metro, Seoul, Korea \\ ${ }^{2}$ School of Robotics, Kwangwoon University, Seoul, Korea
}

Email address:

metro2line@daum.net (Ho-Hung Jung), kslee@kw.ac.kr (Key-Seo Lee)

\section{To cite this article:}

Ho-Hung Jung, Changlong Li, Key-Seo Lee. A Study on the Indoor Positioning Method of a Motorcar Detection System Based on CSS (Chirp Spread Spectrum). Automation, Control and Intelligent Systems. Vol. 3, No. 6, 2015, pp. 133-140. doi: 10.11648/j.acis.20150306.17

\begin{abstract}
Accidents that happen on the Urban Railway or in railroad operating institutions can sometimes involve passengers and often occur with night workers who are carrying out system maintenance and management duties. Various safety related systems and technologies are currently being developed in order to prevent accidents from happening, but it is difficult to find a system that is safe and reliable in regards to unforeseen circumstances of duties carried out at night, on and off the tracks, as well as in tunnels. Despite continuous health and safety education, collision accidents between workers and operating vehicles (motorcars) during maintenance and management at night are regularly occurring, threatening precious lives and rendering the loss of property. The purpose of this paper is to prevent accidents between trains, between motorcars, and between trains and people in the Urban Railway and railroad operating institutions through an RT-ToF (Round Trip Time of Flight), appropriate for vehicles and trains, by developing a device that can trace the distance and location by using an RF module.
\end{abstract}

Keywords: CSS, RT-ToF, Motorcar, Indoor Positioning, RTLS, Positioning

\section{Introduction}

Accidents that happen on the Urban Railway or in railroad operating institutions can sometimes involve passengers and often occur with night workers who are carrying out system maintenance and management duties. Various safety related systems and technologies are currently being developed in order to prevent accidents from happening, but it is difficult to find a system that is safe and reliable in regards to unforeseen circumstances of duties carried out at night, on and off the tracks, as well as in tunnels. Despite continuous health and safety education, collision accidents between workers and operating vehicles (motorcars) during maintenance and management at night are regularly occurring, threatening precious lives and rendering the loss of property.

In order to prevent such accidents, a system that allows the tracing of a location between vehicles (motorcar $\Leftrightarrow$ motorcar) or between a vehicle and a maintenance worker needs to be established through a bilateral detecting system with distance measurement technology along with positioning technology using an RF modulation method.

Object recognition, positioning, action analysis, etc. are in fact key technologies that support an ever- present generation, and as we enter into such a ubiquitous era, the locating system has become quite significant in its position. Nevertheless, existing location services, such as GPS (Global Positioning System), are inappropriate for indoors since their development was designed for the outdoor environment. Consequently, an interest in studies on location systems and the necessities that are compatible for indoors are increasing. Thus, developing a service that allows indoor positioning is essential considering that the service is unsuitable for most consumers who need precise positioning, such as inside a building. Among them, the IEEE 802.15.4a, selected by the UWB (Ultra Wide Band) standard group for low speed, low power, and capacity for wireless positioning, is appropriate for an indoor distance measurement. After considering the processing method of signals that are received from the wireless communication infrastructure and the infrastructure operating technology, etc. when conducting a consecutive indoor and outdoor wireless positioning, the actual method of positioning for the RTLS system, as with GPS and LBS, is classified into three main methods which are, Triangulation, Scene Analysis, and Proximity. Out of these three, locating 
through triangulation is the most superior method and is based on the technologies RSSI (Received Signal Strength Indication) and ToA (Time of Arrival).

Of the two methods, UWB and CSS, that are PHY standards of low speed positioning WPAN, the IEEE 802.15.4a aims to simultaneously achieve the distance measurement with data communication that's based on low power consumption, which is the purpose of IEEE 802.15.4a PHY. With the current UWB base, distance measurement that requires precision within scores of centimeters is actually possible, and with the CSS base, a precise measurement with a $1 \sim 2 \mathrm{~m}$ margin of error is also possible regardless of the distance. Nevertheless, an identical positional value cannot always be calculated due to the characteristics of wireless communication. Therefore, a position correction technique is proposed in this paper in order to increase the accuracy of positioning through major technologies using the RTLS location calculation method.

Diverse methods of bilateral distance measurement have already been currently proposed but so far no case on implementing the performance in underground tunnels has been reported. In this paper however, the application of a positioning system in Urban Railway sections within tunnels was reviewed by applying Ranging and ToA methods using CSS technology, which uses the RF module to measure distance. This CSS (Chirp Spread Spectrum) technology has much better transmission and static characteristics in comparison to those of Zigbee, a standard technology of IEEE 802.15.4, making it suitable for accurate positioning indoors and within short distances.

The CSS (Chirp Spread Spectrum) method and the RT-ToF (Round Trip-Time of Flight) method have been used to measure the actual locations.

\section{Indoor Positioning Method Based on CSS}

\subsection{CSS (Chirp Spread Spectrum)}

CSS technology has been extensively used since 1940 for
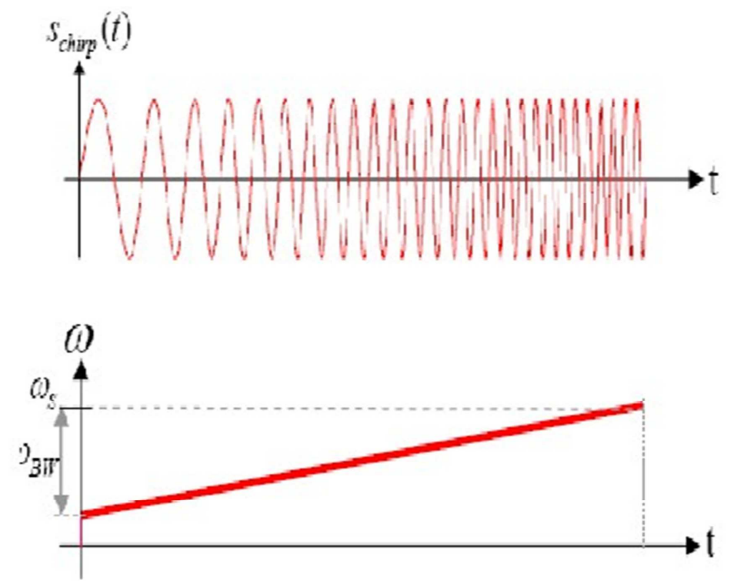

military radar and submarine sound ranging technology, consuming low power while creating powerful radio waves to multi-paths in order to accommodate communication to a broad area. In March 2007, the Institute of Electrical and Electronics Engineers had adopted the CSS technology as one of the physics class technology for the national standard IEEE 802.15.4a, the WPAN standard for indoor wireless communications (IEEE 802.15.4a, 2007). The CSS measures distance through the triangulation method of TOA.

The CSS-based RTLS, which was standardized in the 2007 IEEE 802.15.4a, had showed an enhanced performance in comparison to the existing RSSI and Zigbee method. The Chirp Spread Spectrum aims for tag positioning in a maximum distance of $300 \mathrm{~m}$ with margin of error within a $1 \mathrm{~m}$ radius, through a data-rate of $1 \mathrm{Mbps}$ and $10 \mathrm{dBm}$ transmission power by using CSS signals through a Bandwidth of $22 \mathrm{MHz}$ at $2.4 \mathrm{GHz}$ ISM-Band.

\section{CSS Characteristics.}

Chirp Pulses are a transmission type of technology that implements communication through Pulse signals, which are interference resistant, after they are generated as radio waves increase or decrease in accordance to time. The interaction formula of the changing radio waves is as follows.

$$
S_{c p}(t)=\operatorname{Re}\left[\begin{array}{c}
\operatorname{exd}\left[j\left(\omega_{s}+\frac{\omega_{B W}}{2 T_{c p}} t\right) t+\theta_{0}\right] \\
\times\left[u(t)-u\left(t-T_{c p}\right)\right]
\end{array}\right]
$$

Each composition is as follows.

$T_{c p}$ : Duration of the Linear Chirp Signal.

$\omega_{s}$ : The starting pulse for the start of the Linear Chirp's sweeping.

$\omega_{B W}$ : Sweeping Bandwidth.

$\mathrm{u}(\mathrm{t})$ : Unit Step Function.

The Chirp signal changes to a consistent inclination while the phase changes at a faster pace as time passes, as the above formula is composed of the duration of the Linear Chirp signal, the start pulse at the start of the Linear Chirp's sweeping, the sweeping bandwidth, and the unit step function.
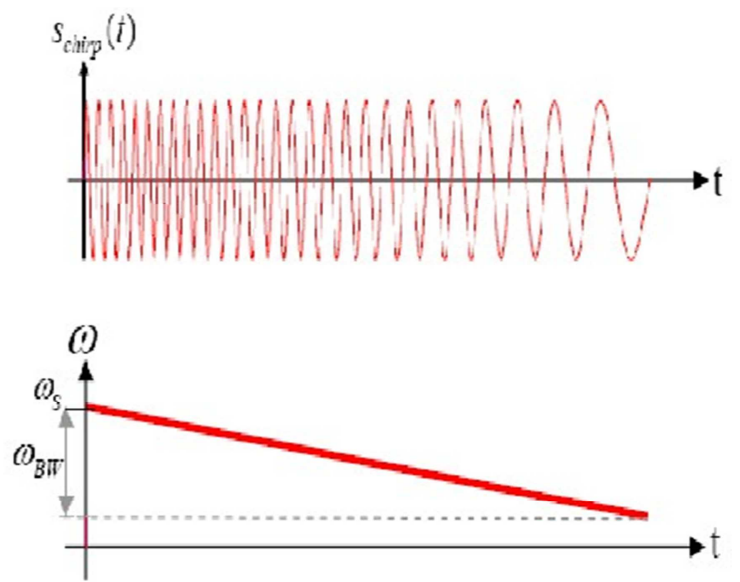

Fig. 1. Chirp Pulse Signals and Frequency Conversion. 
The CSS method is capable to implement a positioning margin of error within $1 \mathrm{~m}$, the target value, even in an indoor environment since the direct waves and multi-path signals can be separated in the receiver by de-correlation, including even the multi-path signals with a path difference within $1 \mathrm{~m}$, due to the physical characteristics of the Chirp signal. The advantages of the CSS method is that it allows a long-distance transmission as it is resistant to confusion and multi-path fading since it employs Chirp pulse signals with the compressed power of Chirp pulses that is spread across a broad bandwidth. As well as that the system can be formed with a low power consumption due to Chirp signal generation using DDL that is composed of simple passive devices.

\subsection{RT-ToF (Round Trip-Time of Flight)}

The RT-ToF (Round Trip-Time of Flight) method was originally proposed to solve the problems of ToF and TDoF, as the name demonstrates ToF is a method using a Round Trip.

The ToF method using RTT concludes the time needed for the signal to travel back and forth between tags.

In other words, supposing that the time taken for the radio wave that is generated from the RFID reader to reach an RFID tag, then for the response to the RFID tag to reach the RFID reader is identical as $t_{p r o p}$, and that the time taken for the tag to receive the signal and respond to the reader is $\mathrm{t}_{\text {tag, }}$, then the time it takes for a trip back and forth between the reader and the tag, $t_{\mathrm{RTT}}$, then results as the following formula.

$$
t_{R T T}=2_{\text {prop }}+t_{\text {tag }}
$$

Arranging this into a formula of $\mathrm{t}_{\text {prop }}$ is as follows.

$$
t_{\text {prop }}=\frac{t_{R T T}-t_{\text {tag }}}{2}
$$

Here, the $t_{\text {tag }}$ is the value that is necessary for the RFID tag to process data experimentally, while the $t_{R T T}$ measures each signal's a response from the reader. Since the $t_{\text {RTT }}$ needs to be approximately $10 \mu \mathrm{sec}$ in order to provide positioning accuracy of meters, the time information of the RFID reader and $\mu$ sec standard must be provided.

For this, a high-precision oscillator should be used, which acts to increase the price and the size of the circuit.

Aside from this, the downside is that the time it takes to calculate the transmission time of radio waves takes 2 to 6 times more than the original ToF method, so consequently the amount to be calculated increases slightly. Nevertheless, this is minor detail compared to the limitations of modulating the starter.

\section{Distance Measurement Calculation.}

The calculated formula of the distance measurement method using the locating technology, a combination of the above formulas, subsequently sending and receiving radio waves, measuring speed, and referring to the image below, is as follows.
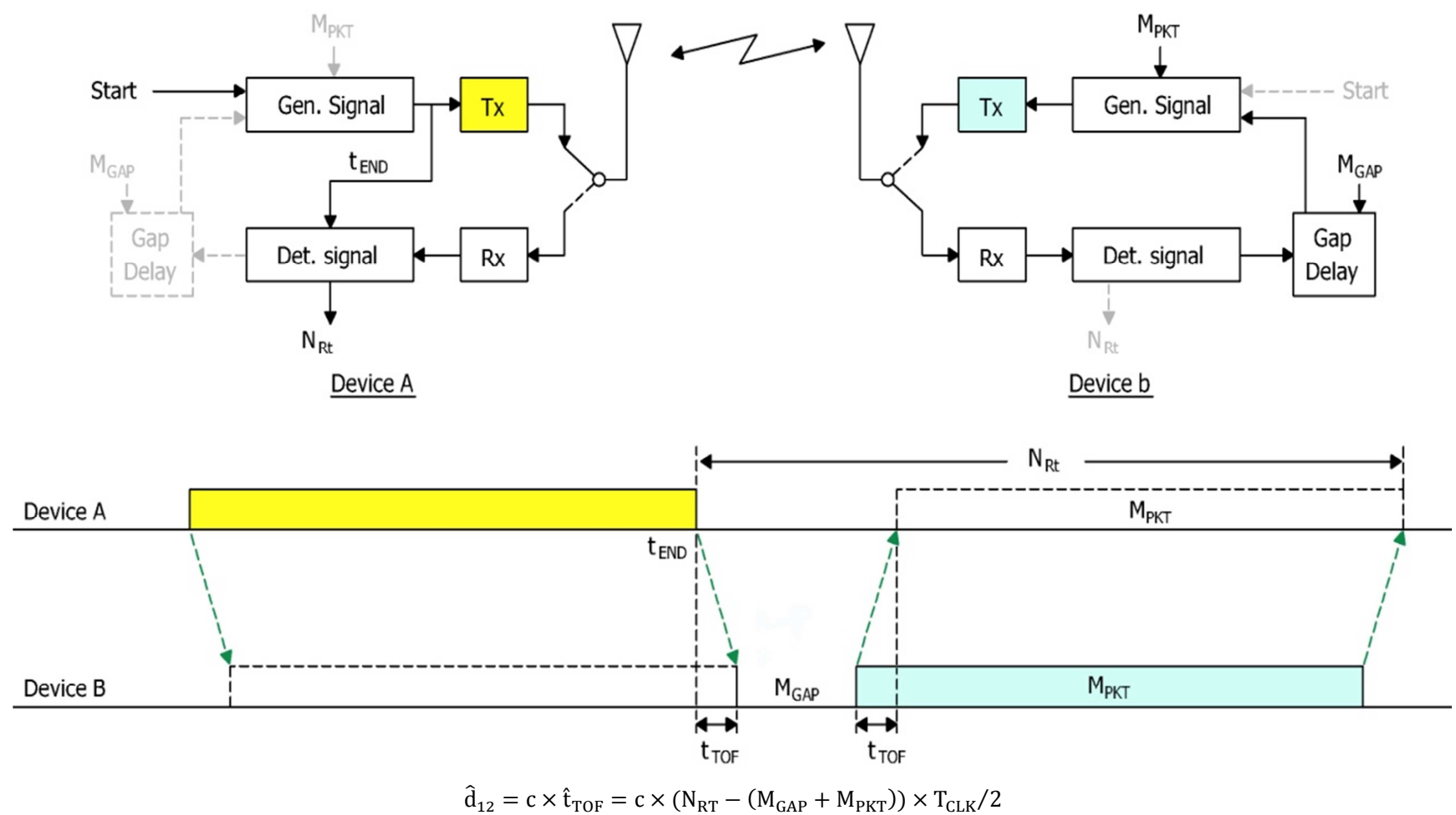

Fig. 2. Concept of the RT-ToF Distance Measurement. 


\subsection{Experiment of Error Correction Filtering Application and Analysis of Results}

\subsubsection{Error Correction Filtering}

The error can only continue to increase if it is difficult to procure LOS between the reader and tag. In particular, the position recognition error increases because of the distance measured through multiple paths, such as the refracted radio waves in a curve section, which cannot be trusted. In order to solve this problem, the error correction filtering was used. The most commonly used algorithm in position recognition systems uses arithmetic mean and the weighted average techniques.

With the arithmetic mean technique, it is problematic to obtain an approximate value since it is simply calculated through the mean when a massive error occurs. Therefore, the error correction filtering was then developed by applying the weighted average technique, which applies the weighted value in accordance to the measured time.

The data filtering method is as follows.

Firstly, when trying to delete a particular range of data, specify the data's minimum and maximum value. Confirm whether the collected data is within the range that is designated by the user, and if the data is outside the range, then return it.

Secondly, there is a specific method that's used when the user wishes to delete any predictable data. Thus, the data transmission number is decreased by deciding whether or not to transmit data after considering the data's margin of error.

The order of the error correction algorithm that was actually implemented is as follows.

1. 10 distance data is measured through $5 \mathrm{~ms}$ of scan time.

2. Of the 10 distance data, detect and delete the minimum value.

3. Of the 10 distance data, detect and delete the maximum value.

4. Calculate the average number from the remaining eight data: (data sum $/ 8=$ avr).

5. Save the 1 time measurement avr data 1 .

6. Collect avr data 2 by measuring the distance from the opposing equipment.

7. Calculation is completed through new_avr $=($ avr data 1 + avr data 2) / 2 .

8. New avr distance data is the valid data, which is automatically saved and displayed on the screen.

Through this process, a more stabilized distance data is calculated.

\subsubsection{Underground Tunnel Site Fixed Point Experiment (Straight Line)}

By applying an error correction filtering system, the tag was measured at Line 9 in the underground tunnel whereby every $10 \mathrm{~m}$ for $10 \sim 50 \mathrm{~m}$ while moving. The Reader System was connected with a motorcar antenna and the Tag System connected with a worker antenna. The below images reveal a comparison from the tags location when applied with only the actual location and the Kit calculation method, with the tag location measured through the data filtering method.

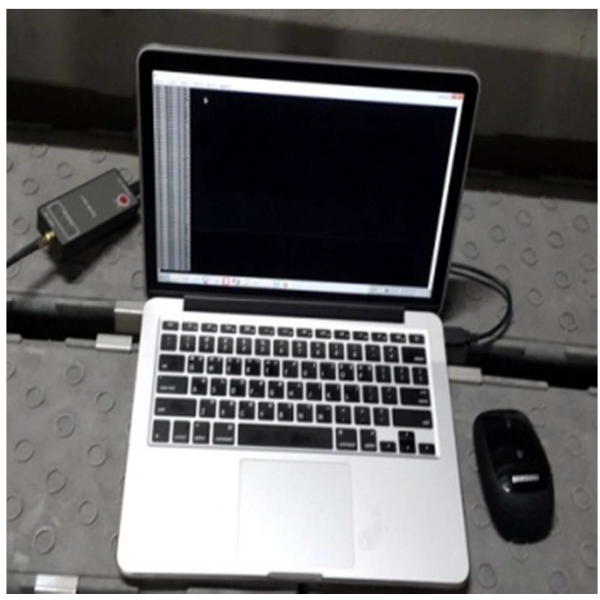

(a) Server System.

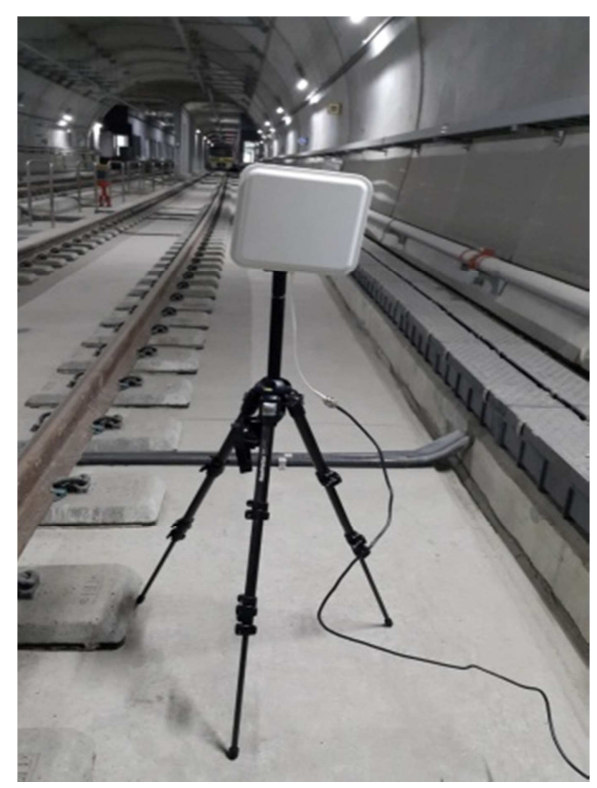

(b) Reader Antenna

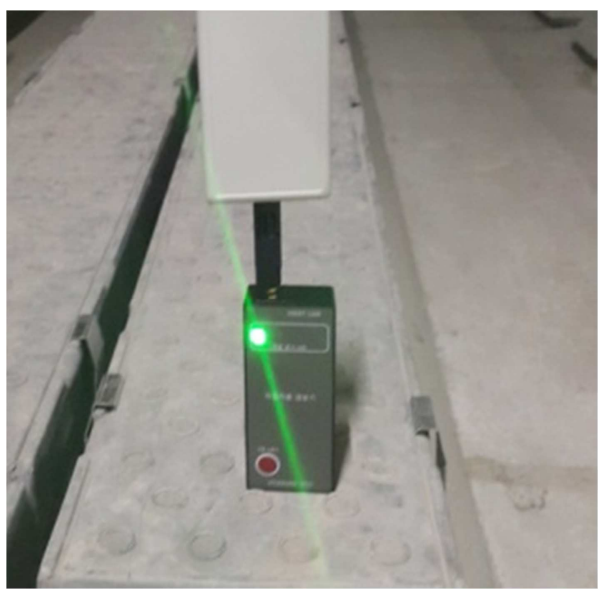

(c) Tag System.

Fig. 3. Site Experiment Photographs. 


\section{Distance $(\mathrm{m})$}

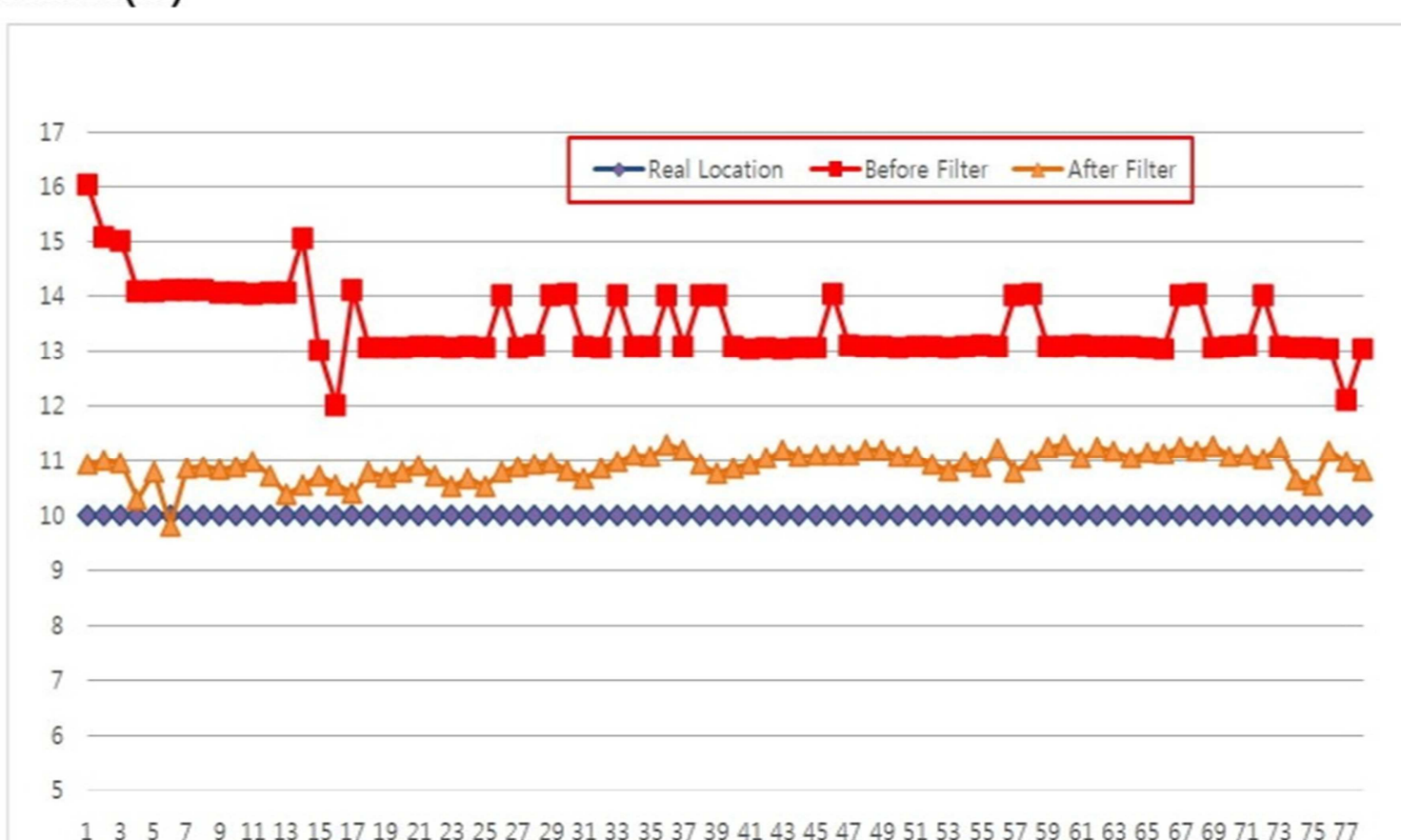

1357911131517192123252729313335373941434547495153555759616365676971737577

Number

(a) $10 \mathrm{~m}$.

\section{Distance $(\mathrm{m})$}

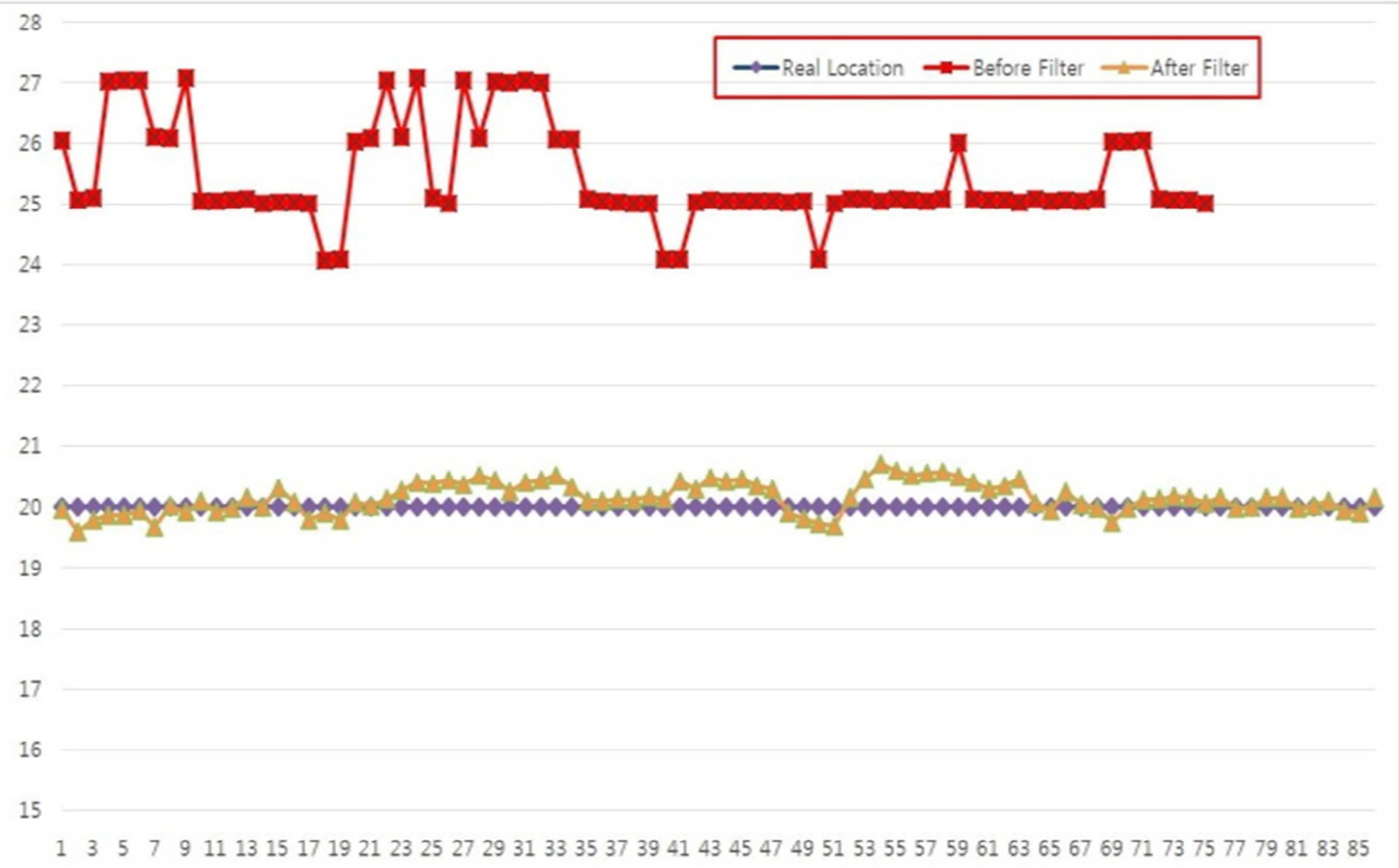

Number

(b) $20 \mathrm{~m}$ 


\section{$\operatorname{Distance}(\mathrm{m})$}

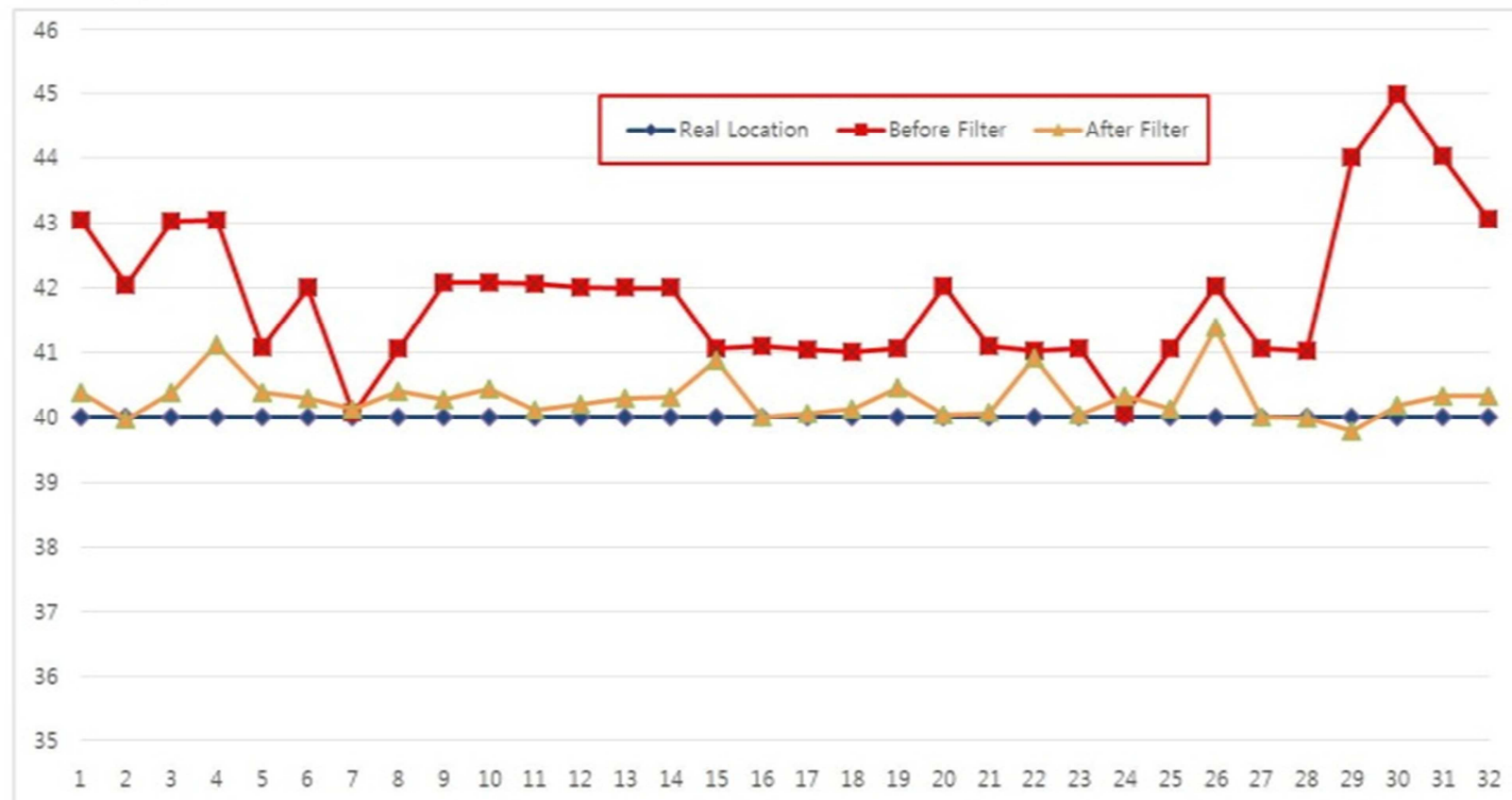

Number

(c) $30 \mathrm{~m}$

\section{Distance $(m)$}

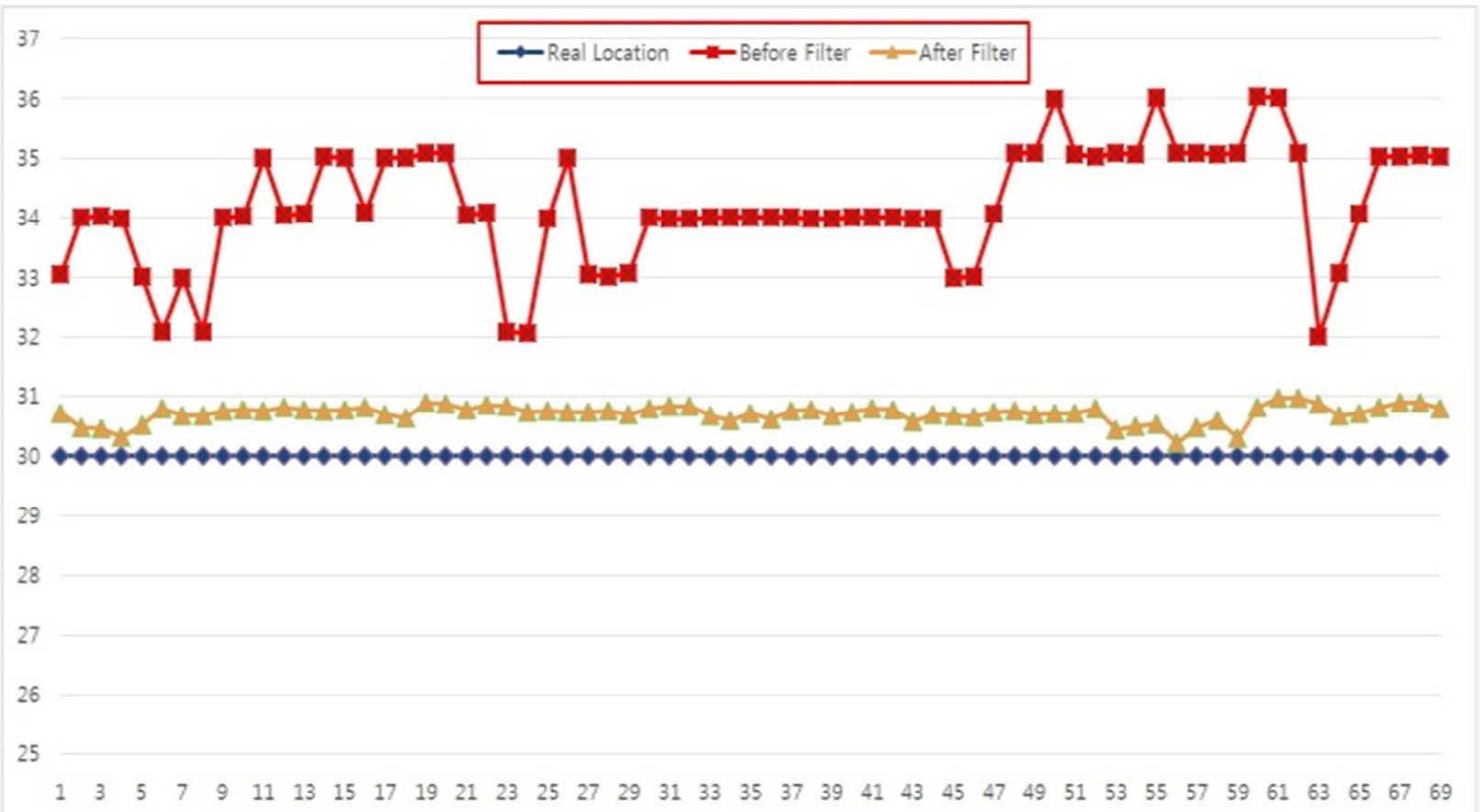

Number

(d) $40 \mathrm{~m}$. 


\section{Distance $(\mathrm{m})$}

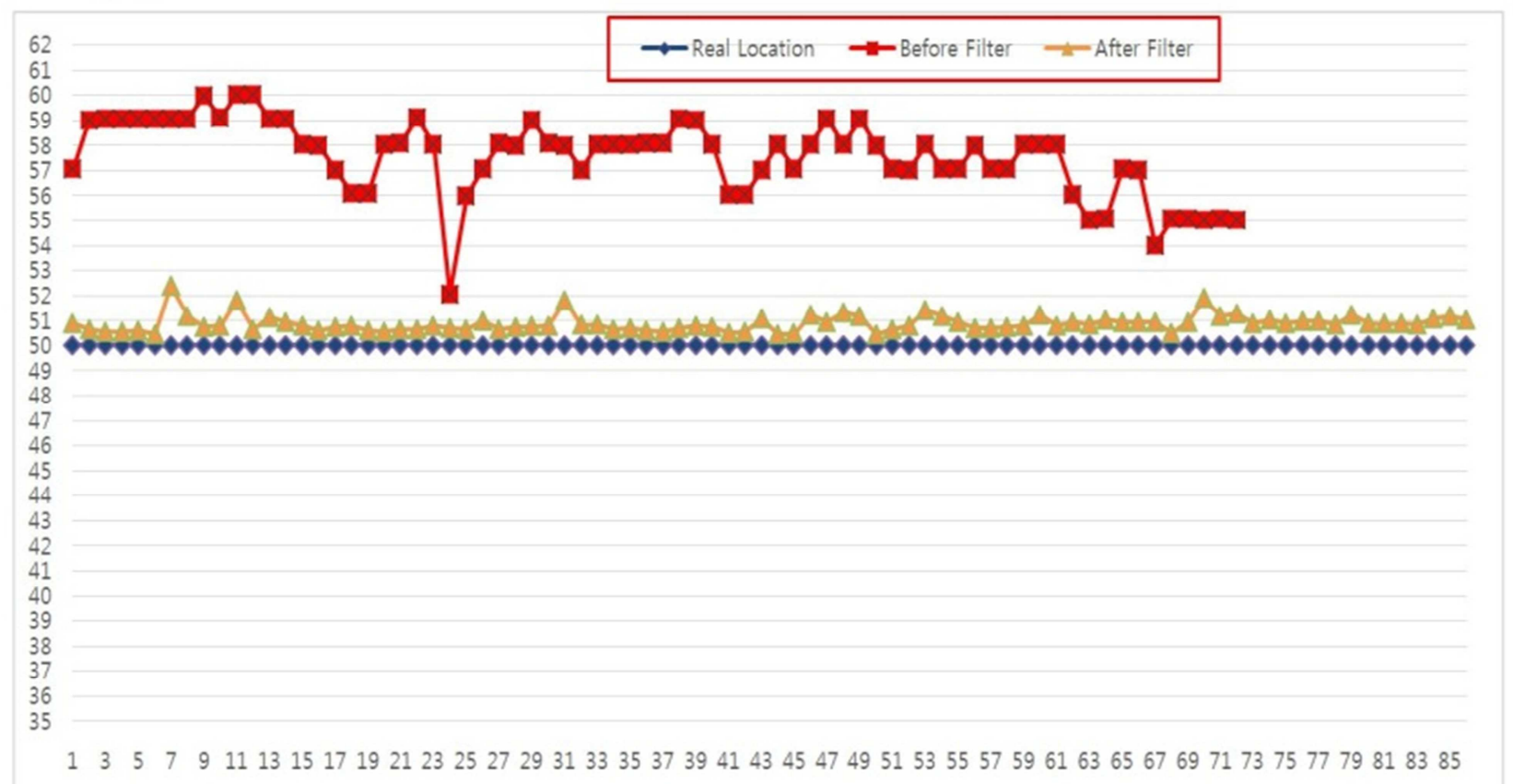

Number

(e) $50 \mathrm{~m}$.

Fig. 4. Measurement distance beforelafter error correction filtering $(10 \mathrm{~m} \sim 50 \mathrm{~m})$.

Fig 4 reveals the comparison of the measurement distance before and after applying the error correction filtering. The blue diamonds are the actual location of the tag, the red squares are the measured location before filtering, and the yellowish green triangles are after the error correction filtering. As displayed in the image, when a high error occurs the error correction filtering is applied, reducing the error to within $2 \mathrm{~m}$.

\subsection{Establishment of a Bilateral Detection System for Urban Railway Maintenance Vehicles}

The bilateral detection system designates the highest address to the exchange frequency that uses CSS to assort internal and external, upper and lower lines and select at the initial operation. When the highest address is designated to the internal (upper) line, the address information on the internal line can be exchanged. In contrast, the external (lower) line can be designated in exactly the same way. The exchange time is analyzed and the distance displayed after the address is designated, and despite the rate of error showing a slight difference according to the speed movement, the goal is to the measure the location $\pm 1 \mathrm{~m}$ when in a stationary condition and $\pm 3 \mathrm{~m}$ when in a motion condition of $45 \mathrm{~km} / \mathrm{h}$.

A system capable of preventing safety accidents was proven by providing the information of an opposing motor within a short-distance having calculated the distance through a RT-ToF method radio wave exchange and displaying the distance.
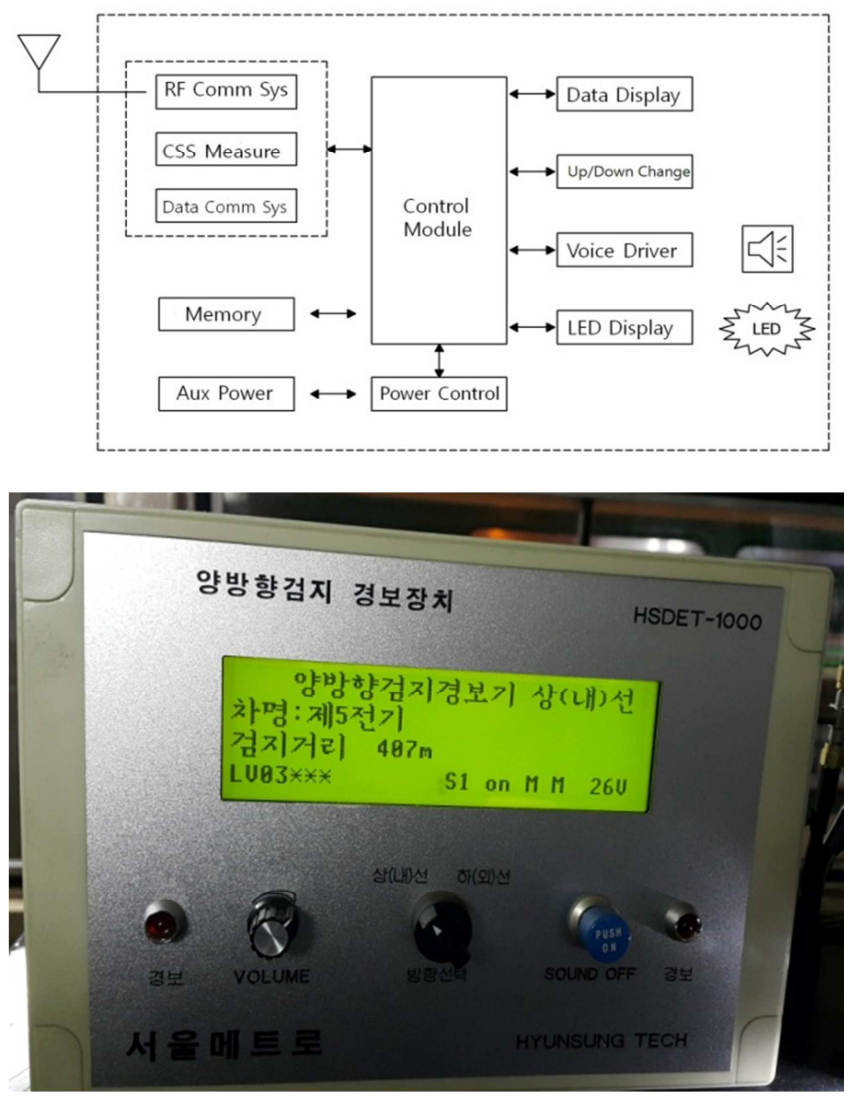

Fig. 5. Bilateral Scanning Alarm Device for Motorcars. 


\section{Conclusion}

In order to realize a motorcar's positioning value or a worker's risk information discovered through RTLS technology, the positioning technology of low cost and high efficiency is necessary.

By reviewing various transmission technologies while comparing and analyzing them the CSS method, which is not affected by existing AP antennas through underground tunnels, was selected as the most appropriate positioning technology for underground sites.

The RTLS technology has several methods for measuring location. The methods include RSS (Received Signal Strength), technology, ToA (Time of Arrival) technology, TDoA (Time Difference of Arrival) technology, AoA (Angle of Arrival) technology, and RT-ToF (Round Trip Time of Flight) technology. After analyzing these different methods the RT-ToF technology was selected as the most appropriate for underground sites.

Lastly, in order to improve the error of position recognition, which is said to be the limitation of the position recognition technology, various error correction algorithms were analyzed and applied. The bilateral detection system for the Urban Railway repair and maintenance car was design and established through the selection of a positioning technology for the position recognition system, selection of a position detection technology for transmitting the position recognition system and various site information, and the selection of an error correction filtering for a correct position recognition.

\section{Acknowledgment}

The present research has been conducted by the research grant of Kwangwoon University in 2013.

\section{References}

[1] Junhyeong Joo, Hyeonuk Kim, Seonghyeon Yang, "The Design and Implementation of a CSS based Location Aware System", Journal of Korea Institute of Information and Communication Engineering, pp. 1549-1558; 2012.

[2] IEEE Standard 802.15.4a-2007: MAC and PHY Spec for Low-rate W-PAN, IEEE.

[3] Daewon Kim (2011) Safety Management System for a Large Construction Project Using Real-time Location System, Doctor's Degree, Korea University.

[4] Yeongho Kim (2011) Adaptive RTLS Algorithm of Passive RFID Tag, Doctor's Degree, Chonnam National University.

[5] Uisang Ahn (2013) Implementation of UWB RTLS System, Master's Degree, Kwangwoon University.

[6] Gyeongguk Lee, "Real Time Location System (RTLS) based on Chirp Signal", Information Processing Society Journal: 2008.

[7] Orthotron, "Chirp 2410 Transceiver" Manual.

[8] Ho-Hung Jung, Changlong Li, Yang - Ok Ko, Key-Seo Lee, "Study on Precise Positioning using Hybrid Track Circuit System in Metro" JKIECS Vol. 8 No. 3, pp. 471-477; 2013.

[9] Deung-Ryeol Yoo, Kyeng-Hwan Hwang, Key-Seo Lee, "A Study on Architecture Design of Output Module for SIL4 Safety Related System", JKIECS Vol. 10. No. 10, pp. 10791086, 2015.

[10] Young-Soo Park, Key-Seo Lee, "A Study on Development of Interlocking Inspection System for Electronic Interlocking System”, JKSR Vol. 5, No. 2, pp 104-111, 2002.

\section{Biography}

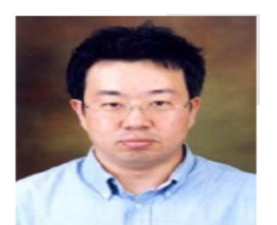

Ho-Hung Jung

1993. 2 Dept. of Control \& Instrument Eng. Kwangwoon Univ. BA.

2015. 8 Dept. of Control \& Instrument Eng. Graduate School of Kwangwoon Univ. Ph. D.

1993 - Seoul Metro, Signal Engineer, Seoul, Korea

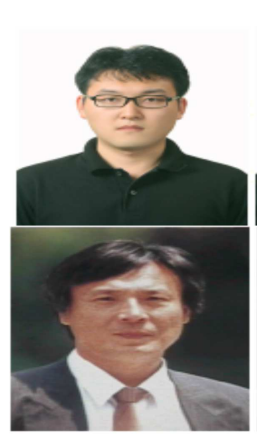

Changlong Li

2008. 2 Dept. of Control \& Instrument Eng. Yantai Univ. China, BA.

2015. 2 Dept. of Control \& Instrument Eng. Graduate School of Kwangwoon Univ. Ph. D.

2016. 3 S-traffic, RAMS engineer, Bundang City, Kyeongki-do

Key-Seo Lee

1970.3-1977.2 Dept. of Electrical Eng. Yonsei Univ. BA.

1977.3-1979.2 Dept. of Electrical Eng. Graduate school of Yonsei Univ. MS.

1979.3-1986.8 Dept. of Electrical Eng. Graduate school of Yonsei Univ. PH. D.

1981.3- School of Robotics, Kwangwoon Univ. Professor. 